\title{
PSR J2021+4026 IN THE GAMMA CYGNI REGION: THE FIRST VARIABLE $\gamma$-RAY PULSAR SEEN BY THE Fermi LAT
}

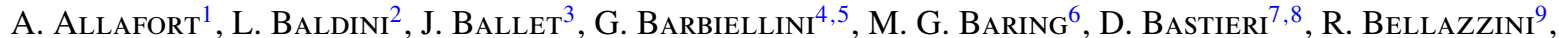
E. Bonamente ${ }^{10,11}$, E. Bottacini ${ }^{1}$, T. J. Brandt ${ }^{12}$, J. Bregeon ${ }^{9}$, P. Bruel ${ }^{13}$, R. BuehleR ${ }^{14}$, S. Buson ${ }^{7,8}$, G. A. Caliandro ${ }^{15}$, R. A. Cameron ${ }^{1}$, P. A. Caraveo ${ }^{16}$, C. Cecchi ${ }^{10,11}$, R.C.G. Chaves ${ }^{3}$, A. Chekhtman $^{17}$, J. Chiang ${ }^{1}$, G. Chiaro ${ }^{8}$, S. Ciprini ${ }^{18,19}$, R. Claus ${ }^{1}$, F. D’Ammando ${ }^{20}$, F. De Palma ${ }^{21,22}$, S. W. Digel ${ }^{1}$, L. Di Venere ${ }^{1}$, P. S. Drell ${ }^{1}$, C. Favuzzi ${ }^{21,22}$, E. C. Ferrara ${ }^{12}$, A. Franckowiak ${ }^{1}$, P. Fusco ${ }^{21,22}$, F. Gargano ${ }^{22}$, D. Gasparrini ${ }^{18,19}$, N. Giglietto ${ }^{21,22}$, M. Giroletti ${ }^{20}$, T. Glanzman ${ }^{1}$,

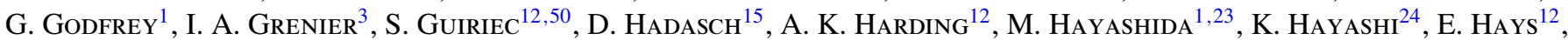

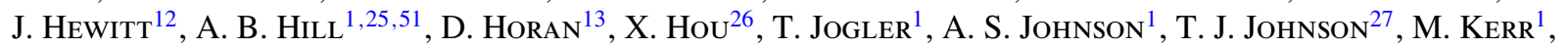

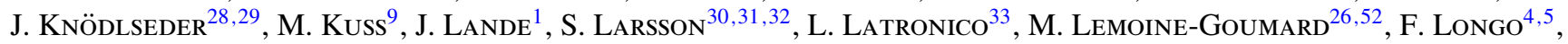

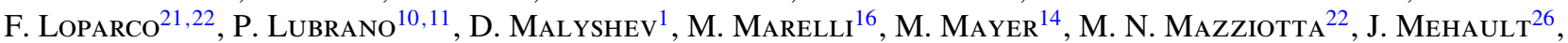
T. Mizuno ${ }^{34}$, M. E. Monzani ${ }^{1}$, A. Morselli ${ }^{35}$, S. Murgia ${ }^{1}$, R. Nemmen ${ }^{12}$, E. Nuss ${ }^{36}$, T. Ohsugi ${ }^{34}$, N. Omodei ${ }^{1}$, M. Orienti $^{20}$, E. Orlando ${ }^{1}$, D. Paneque ${ }^{37,1}$, M. Pesce-Rollins ${ }^{9}$, M. Pierbattista ${ }^{16}$, F. Piron ${ }^{36}$, G. Pivato $^{8}$, T. A. Porter ${ }^{1}$, S. Rainò ${ }^{21,22}$,

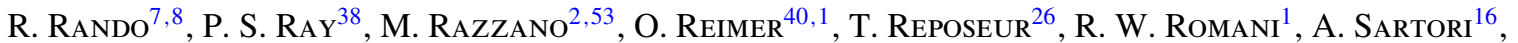

P. M. Saz Parkinson ${ }^{39}$, C. Sgrò ${ }^{9}$, E. J. Siskind ${ }^{41}$, D. A. Smith $^{26}$, P. SPinelli ${ }^{21,22}$, A. W. Strong ${ }^{42}$, H. Takahashi ${ }^{43}$,

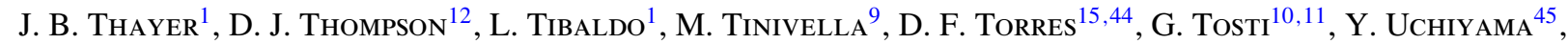
T. L. Usher ${ }^{1}$, J. Vandenbroucke ${ }^{1}$, V. Vasileiou ${ }^{36}$, C. Venter ${ }^{46}$, G. Vianello ${ }^{1,47}$, V. Vitale ${ }^{35,48}$, B. L. WINER ${ }^{49}$, AND K. S. WOOD ${ }^{38}$

${ }^{1}$ W. W. Hansen Experimental Physics Laboratory, Kavli Institute for Particle Astrophysics and Cosmology, Department of Physics and SLAC National Accelerator Laboratory, Stanford University, Stanford, CA 94305, USA; 1tibaldo@ slac.stanford.edu

${ }^{2}$ Università di Pisa and Istituto Nazionale di Fisica Nucleare, Sezione di Pisa I-56127 Pisa, Italy; massimiliano.razzano@pi.infn.it

${ }^{3}$ Laboratoire AIM, CEA-IRFU/CNRS/Université Paris Diderot, Service d'Astrophysique, CEA Saclay, F-91191 Gif sur Yvette, France ${ }^{4}$ Istituto Nazionale di Fisica Nucleare, Sezione di Trieste, I-34127 Trieste, Italy ${ }^{5}$ Dipartimento di Fisica, Università di Trieste, I-34127 Trieste, Italy

${ }^{6}$ Rice University, Department of Physics and Astronomy, MS-108, P. O. Box 1892, Houston, TX 77251, USA

${ }^{7}$ Istituto Nazionale di Fisica Nucleare, Sezione di Padova, I-35131 Padova, Italy

${ }^{8}$ Dipartimento di Fisica e Astronomia “G. Galilei,” Università di Padova, I-35131 Padova, Italy ${ }^{9}$ Istituto Nazionale di Fisica Nucleare, Sezione di Pisa, I-56127 Pisa, Italy

${ }^{10}$ Istituto Nazionale di Fisica Nucleare, Sezione di Perugia, I-06123 Perugia, Italy

${ }^{11}$ Dipartimento di Fisica, Università degli Studi di Perugia, I-06123 Perugia, Italy 12 NASA Goddard Space Flight Center, Greenbelt, MD 20771, USA

${ }^{13}$ Laboratoire Leprince-Ringuet, École polytechnique, CNRS/IN2P3, F-91128 Palaiseau, France

${ }^{14}$ Deutsches Elektronen Synchrotron DESY, D-15738 Zeuthen, Germany

${ }^{15}$ Institut de Ciències de l'Espai (IEEE-CSIC), Campus UAB, E-08193 Barcelona, Spain

${ }^{16}$ INAF-Istituto di Astrofisica Spaziale e Fisica Cosmica, I-20133 Milano, Italy

${ }^{17}$ Center for Earth Observing and Space Research, College of Science, George Mason University, Fairfax, VA 22030, resident at Naval Research Laboratory, Washington, DC 20375, USA

${ }^{18}$ Agenzia Spaziale Italiana (ASI) Science Data Center, I-00044 Frascati (Roma), Italy

${ }^{19}$ Istituto Nazionale di Astrofisica-Osservatorio Astronomico di Roma, I-00040 Monte Porzio Catone (Roma), Italy ${ }^{20}$ INAF Istituto di Radioastronomia, I-40129 Bologna, Italy

${ }^{21}$ Dipartimento di Fisica “M. Merlin” dell’Università e del Politecnico di Bari, I-70126 Bari, Italy

22 Istituto Nazionale di Fisica Nucleare, Sezione di Bari, I-70126 Bari, Italy

${ }^{23}$ Institute for Cosmic-Ray Research, University of Tokyo, 5-1-5 Kashiwanoha, Kashiwa, Chiba, 277-8582, Japan

${ }^{24}$ Institute of Space and Astronautical Science, JAXA, 3-1-1 Yoshinodai, Chuo-ku, Sagamihara, Kanagawa 252-5210, Japan

${ }^{25}$ School of Physics and Astronomy, University of Southampton, Highfield, Southampton, SO17 1BJ, UK

${ }^{26}$ Centre d'Études Nucléaires de Bordeaux Gradignan, IN2P3/CNRS, Université Bordeaux 1, BP120, F-33175 Gradignan Cedex, France

${ }^{27}$ National Research Council Research Associate, National Academy of Sciences, Washington, DC 20001, resident at Naval Research Laboratory, Washington, DC 20375, USA

${ }^{28}$ CNRS, IRAP, F-31028 Toulouse cedex 4, France

${ }^{29}$ GAHEC, Université de Toulouse, UPS-OMP, IRAP, F-31100 Toulouse, France

${ }^{30}$ Department of Physics, Stockholm University, AlbaNova, SE-106 91 Stockholm, Sweden

31 The Oskar Klein Centre for Cosmoparticle Physics, AlbaNova, SE-106 91 Stockholm, Sweden

${ }^{32}$ Department of Astronomy, Stockholm University, SE-106 91 Stockholm, Sweden

${ }^{33}$ Istituto Nazionale di Fisica Nucleare, Sezione di Torino, I-10125 Torino, Italy

${ }^{34}$ Hiroshima Astrophysical Science Center, Hiroshima University, Higashi-Hiroshima, Hiroshima 739-8526, Japan

${ }^{35}$ Istituto Nazionale di Fisica Nucleare, Sezione di Roma "Tor Vergata," I-00133 Roma, Italy

${ }^{36}$ Laboratoire Univers et Particules de Montpellier, Université Montpellier 2, CNRS/IN2P3, F-34095 Montpellier, France

${ }^{37}$ Max-Planck-Institut für Physik, D-80805 München, Germany

${ }^{38}$ Space Science Division, Naval Research Laboratory, Washington, DC 20375-5352, USA

${ }^{39}$ Santa Cruz Institute for Particle Physics, Department of Physics and Department of Astronomy and Astrophysics, University of California at Santa Cruz, Santa Cruz, CA 95064, USA

${ }^{40}$ Institut für Astro- und Teilchenphysik and Institut für Theoretische Physik, Leopold-Franzens-Universität Innsbruck, A-6020 Innsbruck, Austria

${ }^{41}$ NYCB Real-Time Computing Inc., Lattingtown, NY 11560-1025, USA

42 Max-Planck Institut für extraterrestrische Physik, D-85748 Garching, Germany

${ }^{43}$ Department of Physical Sciences, Hiroshima University, Higashi-Hiroshima, Hiroshima 739-8526, Japan

${ }^{44}$ Institució Catalana de Recerca i Estudis Avançats (ICREA), E-08010 Barcelona, Spain

45 3-34-1 Nishi-Ikebukuro,Toshima-ku, 171-8501 Tokyo, Japan

${ }^{46}$ Centre for Space Research, North-West University, Potchefstroom Campus, Private Bag X6001, 2520 Potchefstroom, South Africa 


\author{
${ }^{47}$ Consorzio Interuniversitario per la Fisica Spaziale (CIFS), I-10133 Torino, Italy \\ ${ }^{48}$ Dipartimento di Fisica, Università di Roma “Tor Vergata," I-00133 Roma, Italy \\ 49 Department of Physics, Center for Cosmology and Astro-Particle Physics, The Ohio State University, Columbus, OH 43210, USA \\ Received 2013 July 31; accepted 2013 September 4; published 2013 October 9
}

\begin{abstract}
Long-term monitoring of PSR J2021+4026 in the heart of the Cygnus region with the Fermi Large Area Telescope unveiled a sudden decrease in flux above $100 \mathrm{MeV}$ over a timescale shorter than a week. The "jump" was near MJD 55850 (2011 October 16), with the flux decreasing from $(8.33 \pm 0.08) \times 10^{-10} \mathrm{erg} \mathrm{cm}^{-2} \mathrm{~s}^{-1}$ to $(6.86 \pm 0.13) \times$ $10^{-10} \mathrm{erg} \mathrm{cm}^{-2} \mathrm{~s}^{-1}$. Simultaneously, the frequency spindown rate increased from $(7.8 \pm 0.1) \times 10^{-13} \mathrm{~Hz} \mathrm{~s}^{-1}$ to $(8.1 \pm 0.1) \times 10^{-13} \mathrm{~Hz} \mathrm{~s}^{-1}$. Significant $(>5 \sigma)$ changes in the pulse profile and marginal $(<3 \sigma)$ changes in the emission spectrum occurred at the same time. There is also evidence for a small, steady flux increase over the $3 \mathrm{yr}$ preceding MJD 55850. This is the first observation at $\gamma$-ray energies of mode changes and intermittent behavior, observed at radio wavelengths for other pulsars. We argue that the change in pulsed $\gamma$-ray emission is due to a change in emission beaming and we speculate that it is precipitated by a shift in the magnetic field structure, leading to a change of either effective magnetic inclination or effective current.
\end{abstract}

Key words: gamma rays: stars - pulsars: individual (PSR J2021+4026) - stars: neutron

Online-only material: color figures

\section{INTRODUCTION}

Pulsars are the largest high-energy $\gamma$-ray source class in the Milky Way, with 117 characterized in the second Fermi Large Area Telescope (LAT) pulsar catalog (2PC; Abdo et al. 2013). Early $\gamma$-ray observations suggested glitch-associated pulse and flux changes in the Crab (Greisen et al. 1975) and Vela (Grenier et al. 1988) pulsars, unconfirmed by additional observations (Nolan et al. 2003). Steady $\gamma$-ray fluxes and pulse profiles on timescales longer than those needed for pulsar detections has been an axiom (e.g., Nolan et al. 2012, hereafter 2FGL).

PSR J2021+4026 (hereafter J2021+4026) was discovered in a blind frequency search using LAT data (Abdo et al. 2009). Its spin frequency $f \sim 3.8 \mathrm{~Hz}$ and frequency derivative $\dot{f} \sim-8 \times$ $10^{-13} \mathrm{~Hz} \mathrm{~s}^{-1}$ point to a young, energetic pulsar (characteristic age $\tau_{c}=77 \mathrm{kyr}$, spindown power $\dot{E}_{\mathrm{SD}} \sim 10^{35} \mathrm{erg} \mathrm{s}^{-1}$ ). Radio and optical searches did not yield any plausible counterparts, while deep observations with Chandra and XMM-Newton led to an association with the X-ray source S20 (Weisskopf et al. 2011; Trepl et al. 2010), from which X-ray pulsations were recently detected (Lin et al. 2013). J2021+4026 is seen within the radio shell of the supernova remnant (SNR) G78.2+2.1 (e.g., Ladouceur \& Pineault 2008), also an extended $\gamma$-ray source with $\sim 0$. 6 radius (Lande et al. 2012). A tentative association of these two sources implies a pulsar distance of $\sim 1.5 \mathrm{kpc}$.

Using AGILE observations from 2007 November to 2009 August, Chen et al. (2011) reported variability in 1AGL $\mathrm{J} 2022+4032$, positionally coincident with J2021+4026, but concluded that it was more likely due to another source along the line of sight. This Letter reports the Fermi LAT observation of a discrete change in the $\gamma$-ray flux and frequency derivative of $\mathrm{J} 2021+4026$ and further results from its long-term monitoring.

\section{OBSERVATIONS AND LIKELIHOOD ANALYSIS}

We analyzed $\sim 52$ months of LAT data from 2008 August 4 to 2012 December 11. We selected P7REP_SOURCE "photon"

\footnotetext{
$5^{50}$ NASA Postdoctoral Program Fellow, USA.

${ }^{51}$ Funded by a Marie Curie IOF, FP7/2007-2013-Grant agreement No. 275861 .

${ }^{52}$ Funded by contract ERC-StG-259391 from the European Community.

${ }^{53}$ Funded by contract FIRB-2012-RBFR12PM1F from the Italian Ministry of Education, University and Research (MIUR).
}

class events (Bregeon et al. 2013; Ackermann et al. 2013b) in a $15^{\circ}$ radius Region Of Interest (ROI) with energies from $100 \mathrm{MeV}$ to $300 \mathrm{GeV}$ and zenith angles $\leqslant 100^{\circ}$. We excluded time intervals when the LAT rocking angle was $>52^{\circ}$ or the zone defined by the zenith cut intersected the ROI.

We characterized the spectrum of J2021+4026 through a binned likelihood fit over a $14^{\circ} \times 14^{\circ}$ region centered on the pulsar, with 0.1 angular grid. We used logarithmically spaced energy bins from $100 \mathrm{MeV}$ to $300 \mathrm{GeV}$ (16 below $10 \mathrm{GeV}, 8$ above). The combined likelihood technique (e.g., Ackermann et al. 2012b) treats photons converting in the front and back tracker sections separately to exploit the former's higher angular resolution (Atwood et al. 2009). We used the P7REP_SOURCE_V15 LAT instrument response functions (IRFs) and associated diffuse emission models ${ }^{54}$ and LAT Science Tools v09r32p01. The isotropic background and the residual Earth limb emission were kept fixed. The Galactic diffuse emission was multiplied by a power law with free normalization and spectral index. Our model includes the known extended sources in the region, i.e., the Cygnus- $\mathrm{X}$ cocoon (Ackermann et al. 2011) and SNR G78.2+2.1 (Lande et al. 2012), not included in previous analyses (2FGL, 2PC). We also included 2PC pulsars, and additional 2FGL sources with high significance (average Test Statistic ${ }^{55}$ TS $>100$ ), or within $4^{\circ}$ of J2021+4026. Known flaring sources in the region (Ackermann et al. 2013a) are included in the model. Source spectra were modeled using the functional forms described in the catalogs with all the spectral parameters free. We then searched a TS map of the region for excesses, used as seeds to determine positions and spectra of new sources (see 2FGL). We found five, modeled with log-parabola spectra, at the epoch J2000 positions (R.A., decl.) $=(310.52,42.06),(306.66,40.05)$, (309.65, 42.22), (308.67, 43.05), (312.44, 44.38).

J2021+4026's position was set to Chandra S20's. Its $\gamma$-ray spectrum was modeled using a power law with an exponential cutoff (PLEC; Equation 1), with $b$ free for the phase-averaged spectral analysis and $b=1$ (PLEC1) for the phase-resolved

\footnotetext{
54 The P7REP data, IRFs, and diffuse models (gll_iem_v05.fit, iso_source_front_v05.txt, iso_source_back_v05.txt) will be available at http://fermi.gsfc.nasa.gov/ssc/.

55 The Test Statistic for source detection from maximum likelihood ratio (see, e.g., 2FGL).
} 

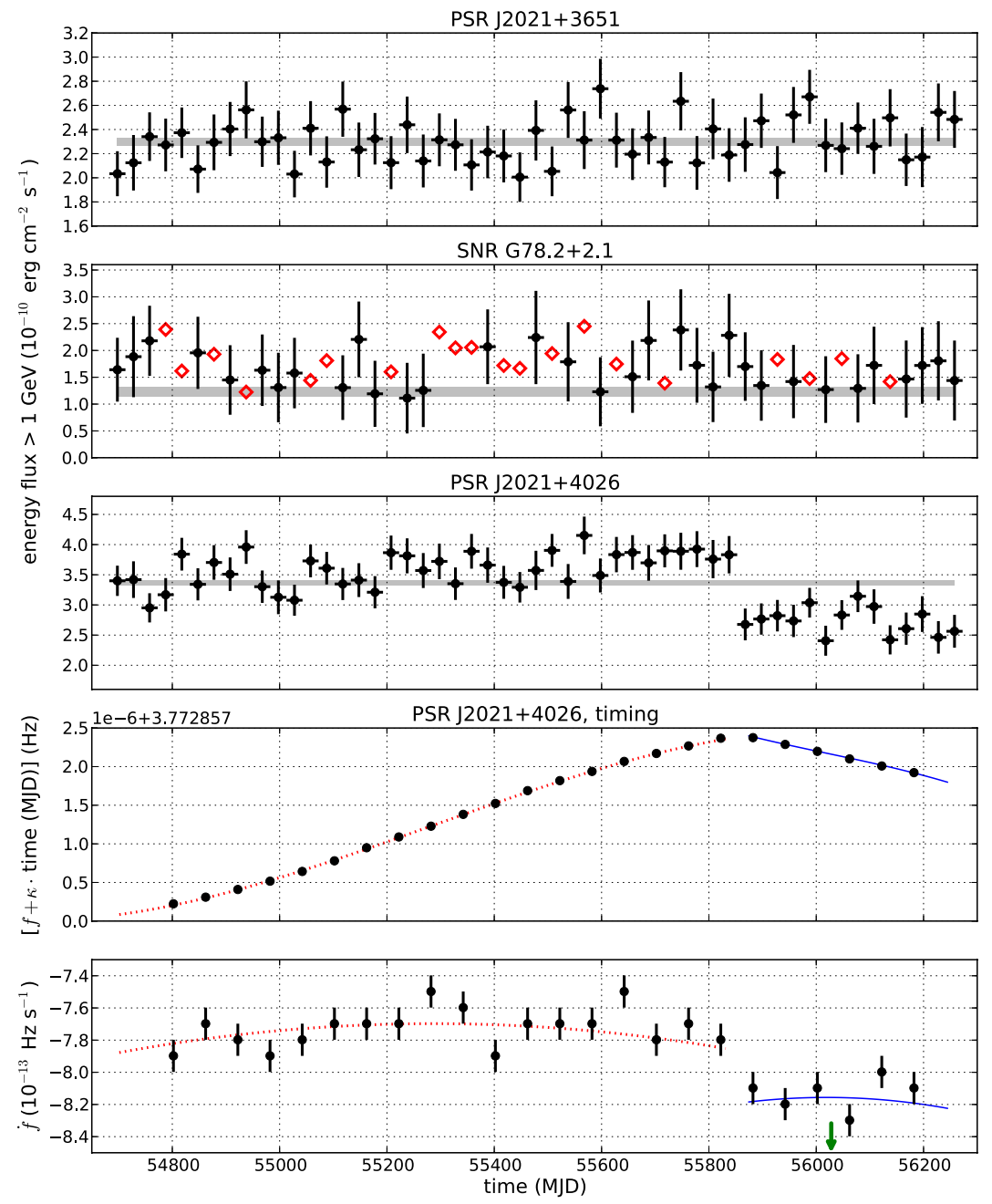

Figure 1. Top three panels: energy flux $(E>1 \mathrm{GeV})$ vs. time in 30 day bins for J2021+3651, SNR G78.2+2.1, and J2021+4026. The gray bands show the average source fluxes for all data. Statistical uncertainties only. We report $95 \%$ confidence level upper limits (red diamonds) for time bins where TS $<4$. Bottom two panels (Section 4): for J2021+4026, $f+\kappa \cdot$ time (MJD), with frequency $f$ and $\kappa=6.9 \times 10^{-8} \mathrm{~Hz}$ day ${ }^{-1}$, and frequency derivative $\dot{f}$, vs. time, from the periodicity search in 60 day windows (points), and from the timing solutions for MJD $<55850$ (red dotted line) and $>55850$ (blue solid line). The green arrow indicates the epoch of the X-ray pulsation detection (Lin et al. 2013).

(A color version of this figure is available in the online journal.)

analysis in Section 5:

$$
\frac{d N}{d E}=N_{0}\left(\frac{E}{E_{0}}\right)^{-\Gamma} \exp \left[-\left(\frac{E}{E_{\mathrm{c}}}\right)^{b}\right] .
$$

PLEC1 represents expectations for high-altitude magnetospheric emission. Phase-averaged spectra are usually better fit with $b<1$ due to the superposition of several PLEC1 components with different photon indices and cutoff energies (Abdo et al. 2010).

\section{FLUX VARIABILITY}

Following Chen et al. (2011), we searched for flux variability around $\mathrm{J} 2021+4026$ at energies $E>100 \mathrm{MeV}$ and $E>1 \mathrm{GeV}$, applying the method in 2FGL. We first fit the data over the entire time range. Then, we divided the range into 7 and 30 day time bins and refit, allowing free normalizations for all sources and fixing the other spectral parameters to their long-term average. The fit was then repeated in each time bin by also fixing the source of interest's normalization to its long-term average. The Galactic diffuse normalization was fit in each time bin, verifying a posteriori its compatibility with a constant. Following 2FGL Equation (4), the fit maximum likelihood values established the probability $P$ that the observed fluctuations are stochastic only. A $2 \%$ flux systematic error accounts for exposure uncertainties between the different epochs.

We applied this procedure to J2021+4026, to SNR G78.2+2.1 (seen in the same direction, but separable from the pulsar at high energies due to its extension ${ }^{56}$ ) and to $\mathrm{J} 2021+3651$, located 3.5 away, with spectrum and flux similar to J2021+4026's. Both SNR G78.2+2.1 and J2021+3651 show constant fluxes $(P>0.4$ in all cases), while J2021+4026 shows significant variability: $P_{30 \text { days }}=7 \times 10^{-8}\left(1 \times 10^{-10}\right), P_{7 \text { days }}=6 \times 10^{-3}\left(2 \times 10^{-4}\right)$ at energies $>100 \mathrm{MeV}(>1 \mathrm{GeV})$.

Figure 1 shows the $>1 \mathrm{GeV}$ energy flux for the three sources in 30 day bins. J2021+4026 shows an abrupt $\sim 20 \%$ flux decrease near MJD 55850 (Table 1), confirmed at energies $>100 \mathrm{MeV}$ and for 7 day bins. We exclude that this drop is due to systematic effects since there is no analogous drop for the two other sources, observed simultaneously. No significant changes in

\footnotetext{
56 The LAT $68 \%$ containment radius for front-converting events is $0.7(<0.2)$
} at 1 (10) GeV (Ackermann et al. 2012a; Bregeon et al. 2013). 
Table 1

J2021+4026's Properties Before and After the Jump

\begin{tabular}{lcc}
\hline \hline $\begin{array}{l}\text { Time Range } \\
\text { (MJD) }\end{array}$ & $54682-55850$ & $55850-56273$ \\
\hline Number of days & 1167 & \\
$F_{\gamma}{ }^{\mathrm{a}}>0.1 \mathrm{GeV}$ & $8.33 \pm 0.08$ & 623 \\
$F_{\gamma}{ }^{\mathrm{a}}>1 \mathrm{GeV}$ & $3.57 \pm 0.05$ & $2.74 \pm 0.13$ \\
$\dot{f}^{\mathrm{b}}$ & $-7.6978 \pm 0.0007$ & $-8.166 \pm 0.002$ \\
$\delta_{\mathrm{P} 1}{ }^{\mathrm{c}}$ & $0.19 \pm 0.02$ & $0.13 \pm 0.02$ \\
$\Delta_{12}{ }^{\mathrm{d}}$ & $0.505 \pm 0.005$ & $0.565 \pm 0.006$ \\
$\delta_{\mathrm{P} 2}{ }^{\mathrm{c}}$ & $0.176 \pm 0.007$ & $0.174 \pm 0.006$ \\
$\Delta_{1 \mathrm{BR}}{ }^{\mathrm{d}}$ & $0.229 \pm 0.008$ & $\ldots$ \\
$\delta_{\mathrm{BR}}{ }^{\mathrm{c}}$ & $0.11 \pm 0.02$ & $\ldots$ \\
$\mathrm{P} 1 / \mathrm{P} 2^{\mathrm{e}}$ & $0.54 \pm 0.06$ & $0.24 \pm 0.03$ \\
$\mathrm{BR} / \mathrm{P} 2^{\mathrm{e}}$ & $0.16 \pm 0.03$ & $\ldots$ \\
$\mathrm{Constant} / \mathrm{P} 2^{\mathrm{e}}$ & $1.83 \pm 0.14$ & $1.09 \pm 0.06$ \\
\hline
\end{tabular}

Notes. Statistical uncertainties only. For details on parameters, see Sections 4 and 5 .

a $10^{-10} \mathrm{erg} \mathrm{cm}^{-2} \mathrm{~s}^{-1}$.

${ }^{\mathrm{b}}$ At the reference epoch for the two timing solutions, $10^{-13} \mathrm{~Hz} \mathrm{~s}^{-1}$.

${ }^{\mathrm{c}}$ Peak FWHM $(E>0.1 \mathrm{GeV})$.

${ }^{\mathrm{d}}$ Phase lag between peaks $(E>0.1 \mathrm{GeV})$.

${ }^{\mathrm{e}}$ Ratios of the peak amplitudes or constant-level-to-P2 amplitude $(E>0.1 \mathrm{GeV})$.

the Fermi observing strategy occurred near MJD 55850. We verified that fixing J2021+4026's normalization to its average yields negative residuals consistent with a point-like source at the pulsar position in all energy bands after MJD 55850.

Figure 1 also suggests a steady flux increase for J2021+4026 before the drop near MJD 55850. A $\chi^{2}$ fit of a linear function of time versus flux in 30 day bins ${ }^{57}$ before MJD 55850 gives a $4 \% \pm 2 \% \mathrm{yr}^{-1}$ flux increase, preferred over the constant flux hypothesis at the $\sim 3.3 \sigma$ level for both $>100 \mathrm{MeV}$ and $>1 \mathrm{GeV}$. The $\chi^{2}$ test applied to $\mathrm{J} 2021+3651$ favors a constant flux. We further assessed this trend independently of any functional dependency using the Kendall rank correlation test. We obtain a Kendall coefficient $\tau=0.78(0.71)$ for J2021+4026 for $>100 \mathrm{MeV}(>1 \mathrm{GeV})$ : the probability ${ }^{58}$ of this coming from stochastic fluctuations of a steady flux is $0.0005(0.01)$, indicating a monotonic increase with time at the $\sim 3.5 \sigma(\sim 3.2 \sigma)$ level. For J2021+3651, $\tau=0.11(0.09)$ : the same probability is $0.38(0.41)$.

\section{PULSAR TIMING}

To investigate the origin of the flux drop, we monitored the evolution of the pulsar timing parameters. We divided the entire time range into 60 day bins, where pulsations are clearly detectable, yet we can neglect the timing noise and approximate the frequency evolution as a linear function,

$$
f(t)=f_{0}+f_{1} \times\left(t-t_{0}\right) .
$$

For each bin, we used the $Z_{n}^{2}(n=4)$ test (Buccheri et al. 1983) to search the $f_{0}-f_{1}$ space for periodicity $\left(f_{0}\right.$ and $f_{1}$ represent the frequency $f$ and frequency derivative $\dot{f}$, respectively, in each bin). Figure 1 shows that near MJD 55850, $\dot{f}$ suddenly decreases

\footnotetext{
57 We assumed a $2 \%$ systematic flux uncertainty, as for the variability test. 58 We take trial factors due to truncating the sample at MJD 55850 into account with a Monte Carlo simulation, where we calculate the maximum $\tau$ for stopping after four different 30 day bins around MJD 55850. The 120 day timescale is independently constrained by the timing analysis in Section 4 (two 60 day bins in the periodicity search).
}

by $\sim 5 \times 10^{-14} \mathrm{~Hz} \mathrm{~s}^{-1}$, i.e., $\sim 4 \%$ of the initial value. This is reflected as a change of the frequency evolution slope, while $f$ does not change appreciably. The $\dot{f}$ change is simultaneous with the flux decrease, strongly suggesting that the flux change is from the pulsar itself rather than another source along the line of sight. This is strengthened by the results for higher energy and narrower time bins (Figure 2), suggesting that the flux variation occurred within a week or less. We also explored three-day and one-day binning, but count rates are too low to measure when and how quickly the flux change occurred. The data hint that it happened within a few days after MJD 55850.

The frequency derivative discontinuity resembles a glitch in $\dot{f}$ (e.g., Cordes \& Downs 1985). However, the phenomenology differs from radio and $\gamma$-ray glitches (e.g., Espinoza et al. 2011; Pletsch et al. 2012): glitches are not usually associated with a flux change and are followed by a recovery, not detected for J2021+4026 prior to MJD 56200.

Doppler shift due to pulsar motion in a binary system cannot explain the change in $f$. If we assume the pulsar moved in the same direction for the $\sim 3$ yr before the jump, i.e., half of a circular orbit with radius 6 (1) a.u., that would yield a fractional frequency change due to the Doppler shift of $10^{-5}\left(10^{-4}\right)$, compared to the observed $\sim 4 \%$ variation. Reproducing the observed change for a six-year orbit requires a highly eccentric orbit with an unrealistically small minor axis of 0.01 a.u. Therefore, the $\dot{f}$ change is likely related to some phenomenon in the pulsar magnetosphere.

The jump causes phase coherence loss. We therefore built two timing solutions using LAT $\gamma$ rays (Ray et al. 2011). We used 32 day intervals to determine pulse times-of-arrival (TOAs). ${ }^{59}$ We obtained 36 (13) before (after) the jump. We used the TEMPO2 package (Hobbs et al. 2006) to fit these TOAs using a model with absolute phase, frequency, and its first three derivatives at the reference epoch. The rms of the timing residuals of the post-jump timing solution is $2.1 \mathrm{~ms}$. The pre-jump solution needed whitening with sinusoidal waves to achieve a $3.0 \mathrm{~ms}$ residual $\mathrm{rms}$. We verified that this is due only to the different lengths of the time ranges. The timing solutions ${ }^{60}$ confirm the sudden $\dot{f}$ change near MJD 55850 (Figures 1 and 2). Owing to similarities with Geminga, we shifted photon phases to center the second highest peak at 0.1 , resulting in a half-period shift relative to $2 \mathrm{PC}$.

\section{PULSAR PROPERTIES BEFORE AND AFTER THE JUMP}

We studied the pulse profile and the spectrum before and after MJD 55850, repeating the likelihood analysis of Section 2 for the two time intervals independently. Then we selected $\gamma$ rays within $2^{\circ}$ of the pulsar and used the best-fit spectrum to assign each photon a weight, the probability of being associated with the pulsar. We assigned each photon a phase using the timing solutions described in Section 4, and thus built weighted pulse profiles for different energy bands (Figure 3). The profiles show two main peaks, P1 and P2, interconnected by a bridge (BR), where a third peak appears before the jump, especially at $E>1 \mathrm{GeV}$. An off-peak region, OP, follows P2.

We fit the pulse profile peaks with three Gaussians, adding a constant to account for steady emission (Figure 3). The third peak in BR is included only when detected with $>3 \sigma$ significance. Table 1 summarizes the fit results and other

\footnotetext{
59 This yields an integer number of TOAs with reasonable pulse profiles.

60 Available at http://fermi.gsfc.nasa.gov/ssc/data/access/lat/ephems/.
} 


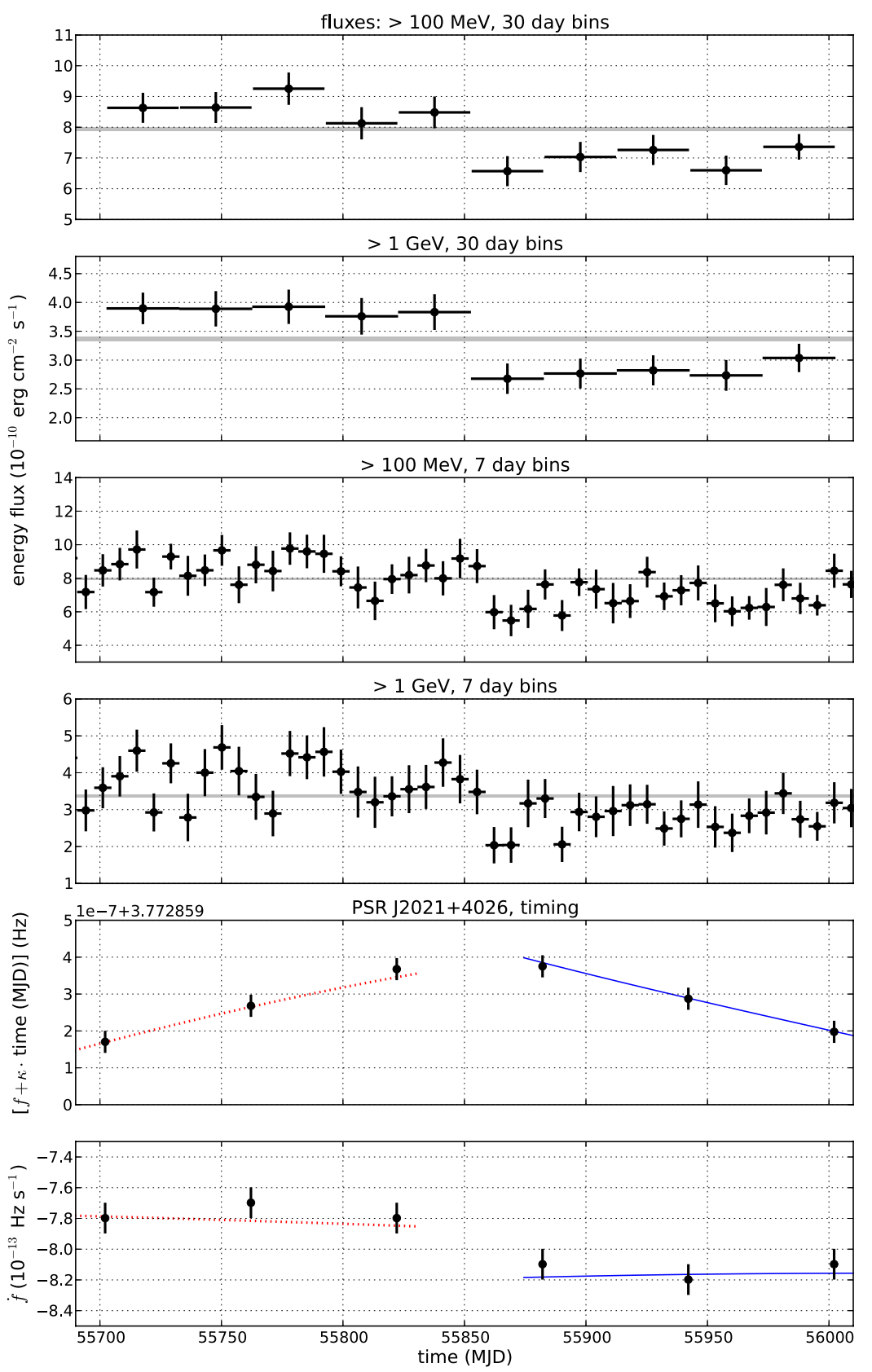

Figure 2. Top four panels: energy flux vs. time for J2021+4026 during 300 days centered at MJD 55850, in different time bins and energy bands. Bottom two panels: $f$ and $\dot{f}$ (described in more detail in Figure 1).

(A color version of this figure is available in the online journal.)

pulsar characteristics. Across the jump the constant component decreases compared to P2's amplitude, and the P1-P2 lag $\left(\Delta_{12}\right)$ increases. The peak height ratio $\mathrm{P} 1 / \mathrm{P} 2$ shows hints of a decrease, while the third peak significance decreases ${ }^{61}$ from $8 \sigma$ to $2.8 \sigma$ $(5.5 \sigma$ to $2.2 \sigma)$ for $>100 \mathrm{MeV}(>1 \mathrm{GeV})$.

To determine the pulsar spectral energy distribution, we subdivided the data before and after the jump into four phase intervals: $0-0.25$ (P1), 0.25-0.4 (BR), 0.4-0.8 (P2), and $0.8-1(\mathrm{OP})$. For each, we determined the pulsar spectrum over 10 logarithmically distributed energy bins from $100 \mathrm{MeV}$ to $10 \mathrm{GeV}$, approximating the spectrum within each bin with a

61 The decrease is partially due to the different epoch lengths before and after the jump. For the 462 days pre-jump, the third peak detection significance decreases to $4.6 \sigma(2.9 \sigma)$ for $>100 \mathrm{MeV}(>1 \mathrm{GeV})$. power law with spectral index 2 . We also determined the spectral energy distribution over the entire energy band using a PLEC1 model.

As shown in Figure 4, across the jump the flux varies at all phases but $\mathrm{P} 2$, strengthening the association of the flux drop with the pulsar as opposed to another source. The OP spectrum is always well-described by a power law with an exponential cutoff at $\sim 2 \mathrm{GeV}$, indicating a magnetospheric origin over all phases. There is an indication of a decrease in $E_{\mathrm{c}}$ for P1 $(\sim 2 \sigma)$.

\section{SUMMARY AND DISCUSSION}

We detected a "jump," a sudden decrease of J2021+4026's flux above $100 \mathrm{MeV}$ of $\sim 20 \%$ associated with a $\sim 4 \%$ increase in spindown rate on a timescale shorter than one week. 

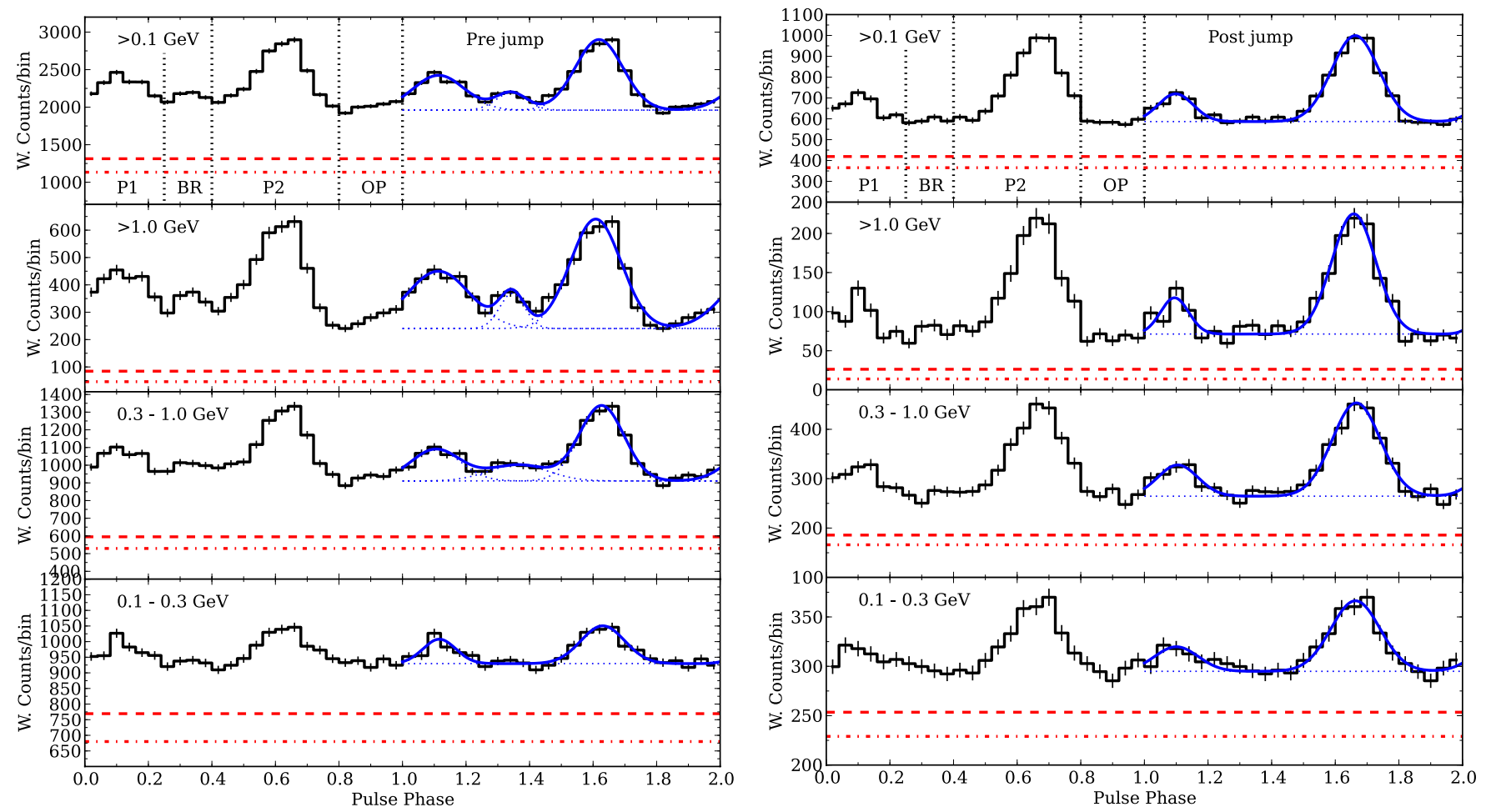

Figure 3. Weighted pulse profiles for J2021+4026 in different energy bands, before (left, 1167 days) and after the jump (right, 423 days). Statistical uncertainties only. Red dashed/dash-dotted line: background level from the spectral fits, including all sources except the pulsar with/without SNR G78.2+2.1. Fit curves overlay the second rotation: blue dotted lines show the constant and Gaussian components, and solid blue lines show the sums.

(A color version of this figure is available in the online journal.)

The jump is also accompanied by changes in the pulse profile. Furthermore, we found evidence for a small, steady flux increase preceding the jump. The temporal correlation between spindown and flux changes strongly indicates that these phenomena are related to the pulsar. While mode changes and other intermittent behavior are well known for some radio pulsars (e.g., Lyne et al. 2010), this is the first time such behavior has been seen at $\gamma$-ray energies.

J2021+4026 belongs to a small set of unusual LAT pulsars-PSR J0633+1746 (Geminga), J1836+5925 and J2021+4026-the sources in 2PC with the brightest magnetospheric emission at all spin phases. They are all radio-quiet, with phase lags between the main peaks $\Delta>0.5$, higher than typical (Abdo et al. 2013). Finally, although only Geminga has a parallax distance (we rely on the SNR association of J2021+4026 and X-ray spectral arguments for J1836+5925), if we adopt the common assumption that the $\gamma$-ray pulse is effectively uniform on the sky, beaming factor $f_{\Omega}=1$, then all three have large efficiencies $\eta=4 \pi f_{\Omega} F_{\gamma} d^{2} / \dot{E}_{\mathrm{SD}} \geqslant 1\left(d\right.$ is the distance and $F_{\gamma}$ is the energy flux; see Table 1). J2021+4026 is the most extreme of the three, with $\eta=2.3$.

All of these attributes point to peculiarity in the $\gamma$-ray beaming. They are most easily understood in the context of the classical outer gap (OG) model. Romani \& Watters (2010) show that such large peak lag implies small magnetic inclinations $\alpha<30^{\circ}$ and near-equatorial viewing angles $80^{\circ}<\zeta<100^{\circ}$. For this geometry the pulsars should be radio-quiet, the $\mathrm{OG}$ geometry predicts $f_{\Omega} \approx 0.1-0.2(\eta<1)$ and the Earth line-ofsight skims nearly tangentially to the peak caustics, producing complex peak structure and strong off-peak emission (Romani \& Watters 2010). Also, two-pole caustic (TPC) models (Dyks $\&$ Rudak 2003) can produce strong off-peak components for a wider range of geometries (most with $\alpha<30^{\circ}$ ). These models tend to have single broad pulses at small $\zeta$, but large $\zeta$ models can be double pulsed. Thus, the preferred geometry is similar to that of the OG case, and should also be radio-quiet. These models have $f_{\Omega} \approx 0.5-0.75$, making it harder to accommodate the observed $\gamma$-ray flux. If classical TPC solutions are extended to higher altitude, then one may recover the broad equatorial pulses and small $f_{\Omega}$ (M. Pierbattista et al. 2013, in preparation). The nearly aligned rotator viewed at high inclination scenario is independently confirmed for Geminga due to X-ray observations of its rotating hot spot (Caraveo et al. 2004).

When emission from near the light cylinder dominates the pulse, the concentration of the $\gamma$-ray beam to a narrow equatorial strip gives high apparent $\eta$ and allows small changes in magnetic field morphology or even in $\alpha$ to move a substantial fraction of the $\gamma$-ray beam over the line of sight, giving large fractional changes to the pulse profile and $f_{\Omega}$. For young pulsars, we expect the $\gamma$-ray luminosity to scale with $\sqrt{\dot{E}_{\mathrm{SD}}}$ (e.g., Harding 1981; Abdo et al. 2013). The decrease in flux rate associated to an increase in spindown rate after the jump strengthens the case that beaming must play a key role.

Therefore, we can speculate that the jump of J2021+4026 represents a shift in the magnetic field structure, leading to either an effective $\alpha$ change or an effective current change. These may be precipitated by a reconfiguration of field line footpoints at the surface, i.e., in the crustal layers, that modifies the overall magnetic dipole torque on the star. There is no reason to expect that the resulting spindown increase should enhance the solidangle integrated luminosity of the pulsar $\gamma$-ray emission, since the principal effect is that of a modified beaming. If the slow variation in the pulsar flux before the jump is substantiated by additional study, this might plausibly be associated with 

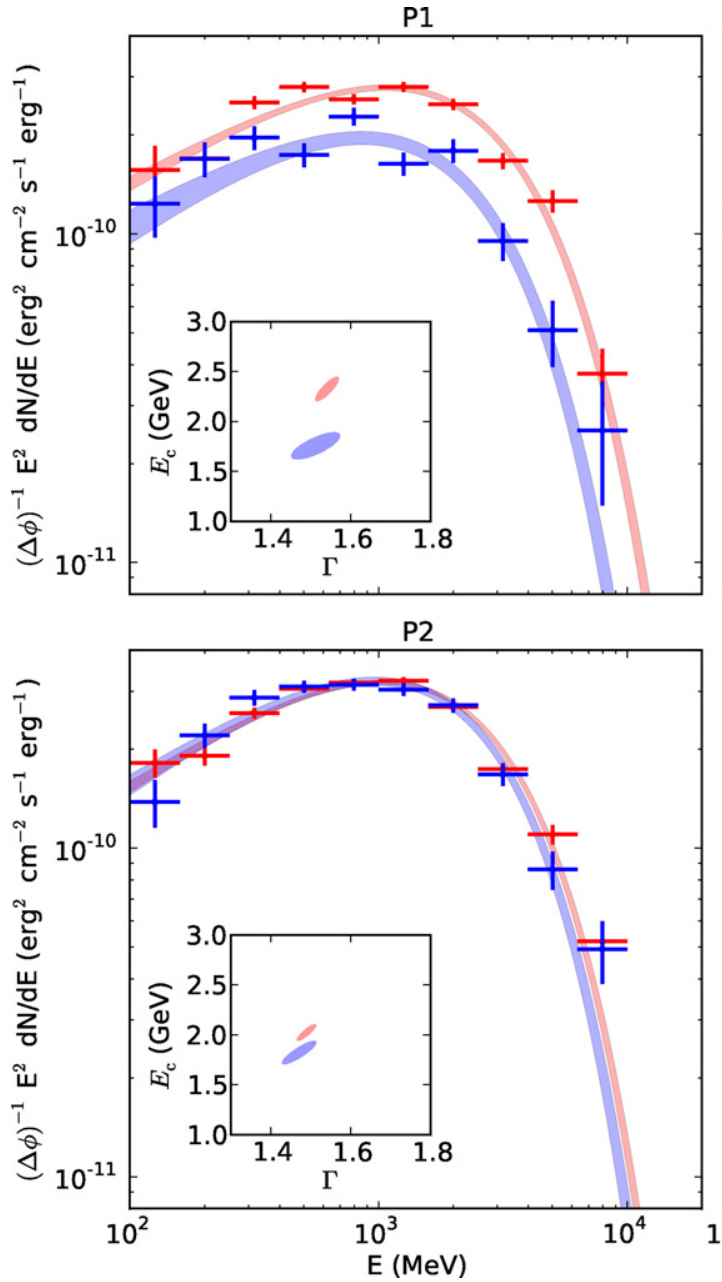

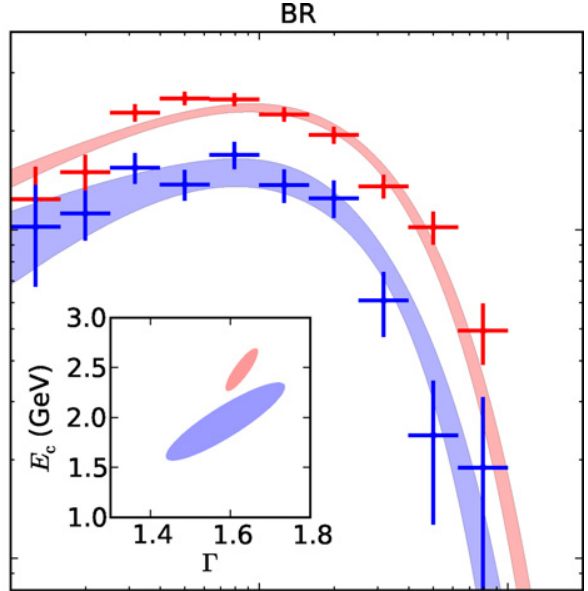

OP

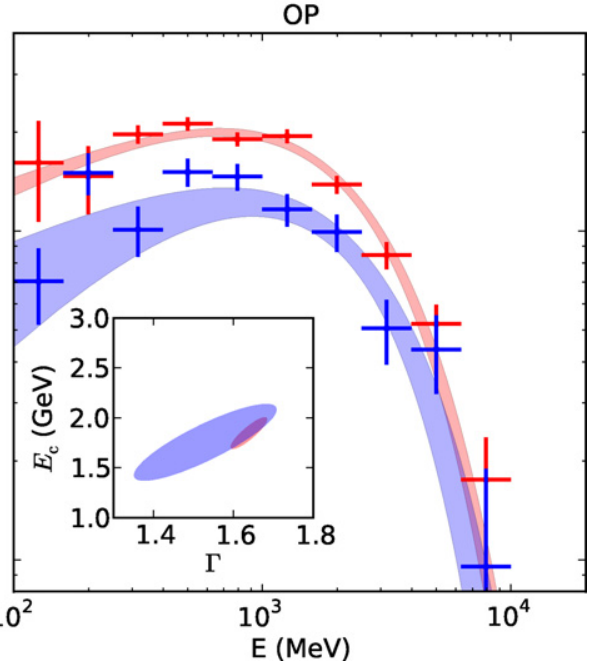

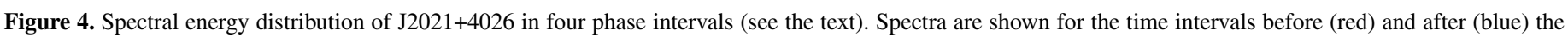

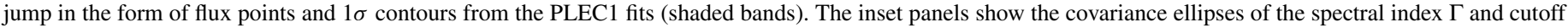
energy $E_{c}$ for the best-fit PLEC1 model. Statistical uncertainties only.

more gradual changes in geometry, for example, from forcefree precession (e.g., Jones 2012).

The very sensitivity of the beaming to currents and geometry for the equatorial, small $\alpha$, OG, or TPC models complicates the interpretation of the observations in terms of magnetosphere configurations. Alternative tests of these scenarios may rely on non- $\gamma$-ray constraints on spin geometry, e.g., from X-ray imaging of the synchrotron termination shock ( $\mathrm{Ng} \&$ Romani 2004), or, if radio-loud examples can be detected, from polarization studies.

Radio and X-ray observations have shown that mode changes, and variability in general, are key to understanding pulsars (Lyne et al. 2010; Hermsen et al. 2013) and therefore to investigating their fundamental physics (Alpar et al. 1984; Cordes et al. 2004). The "jump" in J2021+4026 breaks the axiom of pulsars as steady $\gamma$-ray emitters, opening new avenues for pulsar variability studies at $\gamma$-ray energies, where the bulk of their spindown energy is emitted.

The Fermi LAT Collaboration acknowledges support from a number of agencies and institutes for both the development and the operation of the LAT as well as scientific data analysis. These include NASA and DOE in the United States, CEA/Irfu and IN2P3/CNRS in France, ASI and INFN in Italy, MEXT, KEK, and JAXA in Japan, and the K. A. Wallenberg Foundation, the Swedish Research Council, and the National Space Board in Sweden. Additional support from INAF in Italy and CNES in France for science analysis during the operations phase is also gratefully acknowledged.

\section{REFERENCES}

Abdo, A. A., Ackermann, M., Ajello, M., et al. 2009, Sci, 325, 840 Abdo, A. A., Ackermann, M., Ajello, M., et al. 2010, ApJ, 713, 154 Abdo, A. A., Ajello, M., Albert, A., et al. 2013, ApJ, in press (arXiv:1305.4385) (2PS)

Ackermann, M., Ajello, M., Albert, A., et al. 2012a, ApJS, 203, 4 Ackermann, M., Ajello, M., Albert, A., et al. 2013a, ApJ, 771, 57

Ackermann, M., Ajello, M., Albert, A., et al. 2013b, PhRvD, submitted (arXiv:1305.5597)

Ackermann, M., Ajello, M., Allafort, A., et al. 2011, Sci, 334, 1103

Ackermann, M., Ajello, M., Allafort, A., et al. 2012b, Sci, 338, 1190

Alpar, M. A., Pines, D., Anderson, P. W., \& Shaham, J. 1984, ApJ, 276, 325

Atwood, W. B., Abdo, A. A., Ackermann, M., et al. 2009, ApJ, 697, 1071

Bregeon, J., Charles, E., \& Wood, M., for the Fermi-LAT Collaboration 2013, in Proc. 2012 Fermi Symposium, ed. N. Omodei, T. J. Brandt, \& C. WilsonHodge, eConf C121028, arXiv:1304.5456

Buccheri, R., Bennett, K., Bignami, G. F., et al. 1983, A\&A, 128, 245

Caraveo, P. A., De Luca, A., Mereghetti, S., Pellizzoni, A., \& Bignami, G. F. 2004, Sci, 305, 376

Chen, A. W., Piano, G., Tavani, M., et al. 2011, A\&A, 525, A33

Cordes, J. M., \& Downs, G. S. 1985, ApJS, 59, 343

Cordes, J. M., Kramer, M., Lazio, T. J. W., et al. 2004, NewAr, 48, 1413

Dyks, J., \& Rudak, B. 2003, ApJ, 598, 1201 
Espinoza, C. M., Lyne, A. G., Stappers, B. W., \& Kramer, M. 2011, MNRAS, 414, 1679

Greisen, K., Ball, S. E., Jr., Campbell, M., et al. 1975, ApJ, 197, 471

Grenier, I. A., Hermsen, W., \& Clear, J. 1988, A\&A, 204, 117

Harding, A. K. 1981, ApJ, 245, 267

Hermsen, W., Hessels, J. W. T., Kuiper, L., et al. 2013, Sci, 339, 436

Hobbs, G. B., Edwards, R. T., \& Manchester, R. N. 2006, MNRAS, 369,655

Jones, D. I. 2012, MNRAS, 420, 2325

Ladouceur, Y., \& Pineault, S. 2008, A\&A, 490, 197

Lande, J., Ackermann, M., Allafort, A., et al. 2012, ApJ, 756, 5
Lin, L. C. C., Hui, C. Y., Hu, C. P., et al. 2013, ApJL, 770, L9

Lyne, A., Hobbs, G., Kramer, M., Stairs, I., \& Stappers, B. 2010, Sci, 329, 408 Ng, C.-Y., \& Romani, R. W. 2004, ApJ, 601, 479

Nolan, P. L., Abdo, A. A., Ackermann, M., et al. 2012, ApJS, 199, 31 (2FGL) Nolan, P. L., Tompkins, W. F., Grenier, I. A., \& Michelson, P. F. 2003, ApJ, 597,615

Pletsch, H. J., Guillemot, L., Allen, B., et al. 2012, ApJ, 744, 105

Ray, P. S., Kerr, M., Parent, D., et al. 2011, ApJS, 194, 17

Romani, R. W., \& Watters, K. P. 2010, ApJ, 714, 810

Trepl, L., Hui, C. Y., Cheng, K. S., et al. 2010, MNRAS, 405, 1339

Weisskopf, M. C., Romani, R. W., Razzano, M., et al. 2011, ApJ, 743, 74 